\title{
Forces in a true and physical sense: From mathematical models to metaphysical conclusions
}

\begin{abstract}
J. Wilson (2009), Moore (2012), and Massin (2017) identify an overdetermination problem arising from the principle of composition in Newtonian physics. I argue that the principle of composition is a red herring: what's really at issue are contrasting metaphysical views about how to interpret the science. One of these views - that real forces are to be tied to physical interactions like pushes and pulls - is a superior guide to real forces than the alternative, which demands that real forces are tied to "realized" accelerations. Not only is the former view employed in the actual construction of Newtonian models, the latter is both unmotivated and inconsistent with the foundations and testing of the science.
\end{abstract}

\section{Introduction}

The metaphysical interpretation of Newtonian mechanics is almost as old as the theory itself. One of the questions that arises in this domain is which forces we ought to believe in, given the assumption that a force-based interpretation of Newtonian mechanics is the right one. The philosophical literature has identified two candidates for the "real" (or causally efficacious) forces: the total or "resultant" forces, preferred due to their close connection with acceleration, and the forces that represent particular physical interactions (called 
"component forces"), preferred due to their presence in specific force laws like the law of gravity. ${ }^{1}$ These two types of forces are related by the principle of composition, which says that the latter sum together to produce the former, and J. Wilson (2009), Moore (2012), and Massin (2017) have thus argued that Newtonian mechanics faces an overdetermination problem, as both types of forces are sufficient for the generation of observed accelerations.

My interest in this debate is more interpretative than metaphysical: the question provides the opportunity for an interesting case study in how the formulations or applications of a science can provide evidence for particular metaphysical interpretations of that science. To this end, I focus on the historical foundations and practical uses of Newtonian physics: what metaphysical commitments do these features of the science support, and which do we impress upon them for other reasons? I argue for three main theses. First, that force composition, which serves as the explicit motivation for the prior literature, is a red herring: what really drives the overdetermination problem are metaphysical assumptions brought to the interpretation of Newtonian mechanics, not the theory itself $(\S 1)$. Second, though Newtonian mechanics is not incompatible with a metaphysical view in which total forces are causally efficacious, it gives us no reason to believe in such a view: the argument from features of accelerations to features of forces can't be supported on the basis of the science itself, and may in fact be undermined by it (§2). Third, and by contrast, there are successful applications of Newtonian mechanics in which the connection between real forces and physical interactions like collisions or gravitational attractions plays an essential role in the construction or testing of a model, and these applications do give us reason to believe that

\footnotetext{
${ }^{1} \mathrm{I}$ 'm adopting the language of "real" forces from the literature. In my mouth, at least, this means solely that the forces in question are causally efficacious, that they are - in Newton's terms - "true and physical" (Newton 1726/1999, 408) as opposed to mathematical vectors that represent such forces. I have no intention of trying to determine whether mathematical objects such as vectors are "real" in some important sense and don't deny that there will be cases in which the best explanation involves the total force acting on a body. My point, in declaring some elements of Newtonian physics real and others not, is to demarcate which of these elements are taken to have causal implications of their own and which represent or encode the effects of the former.
} 
the forces that represent these interactions are real $(\S 3)$.

As a result, I argue - with Creary (1981) and against much of the subsequent literaturethat given the assumption of a force-based interpretation, the causally efficacious elements of the world are the forces that appear in the special force laws rather than the total forces. Dialectically, my results put accounts like J. Wilson (2009)'s, Moore (2012)'s, or Massin (2017)'s in a vice: if I'm right, there are no broadly-speaking interpretative reasons to believe that total forces are causally efficacious, and so any account that postulates their causal efficacy must do so on a priori metaphysical grounds alone. ${ }^{2}$ Supposing that such a priori metaphysical grounds are forthcoming and thus that there are in fact good reasons to believe in the causal efficacy of both physical interactions and total forces, however, the resulting metaphysical problems seem (to me, at least) either to motivate rejecting the assumption that the best interpretation of Newtonian mechanics is force-based or to support the unsurprising conclusion that there are deep conceptual issues with Newtonian mechanics related to how it handles inertia. In other words, if my arguments are successful, views on which total forces are real have no serious interpretative advantage over a metaphysics based on Newton-Cartan theory (say), and must therefore motivate opting for the former rather than the latter on non-interpretative grounds.

\section{The physics and metaphysics of forces}

Consider a simple Newtonian model of our solar system. Given measurements of the masses $\left(m_{i}\right)$ for each of the bodies $(i)$ in the system $(S)$ and a measurement of the distance between each pair of bodies $\left(r_{i j}\right)$, Newtonian physics will represent their interaction using a pair of

\footnotetext{
${ }^{2}$ J. Wilson (2009) also gives an experiential or phenomenological argument in support of total forces. I take it that the same considerations apply.
} 
force vectors with the following relationship:

$$
F_{i j}=G \frac{m_{i} m_{j}}{\left(r_{i j}\right)^{2}}\left\langle\hat{x}_{j-i}, \hat{y}_{j-i}, \hat{z}_{j-i}\right\rangle=-F_{j i}
$$

where $F_{i j}$ should be read as the the force from $j$ on $i$ and $\left\langle\hat{x}_{j-i}, \hat{y}_{j-i}, \hat{z}_{j-i}\right\rangle$ is the unit vector leaving $j$ in the direction of $i$. The standard textbook procedure for predicting motion in Newtonian physics proceeds by setting these force vectors equal to acceleration vectors by way of Newton's second law. These accelerations are then used (in combination with initial conditions) to derive velocities and displacements.

Of particular import is the fact that the effects of different force vectors on a single body combine linearly: there is nothing more to their joint effect than the separate effects added together. This fact is expressed by the "principle of composition of forces." As a consequence of the principle, a model in which only a single force, $F_{i}$, acts on $i$ will predict the same acceleration for $i$ as the first model if and only if $F_{i}$ is the vector sum of all the forces in the first model, i.e. iff

$$
F_{i}=\sum_{j \in S} F_{i j}
$$

Of course, the principle of composition says more than this: any models with the same vector summation of forces acting on $i$ will predict the same acceleration for $i$. In highly anachronistic terms, we could say that the principle defines a class of transformations under which the acceleration of a single body is invariant. ${ }^{3}$

The principle of composition alone is metaphysically unproblematic. There's nothing

\footnotetext{
${ }^{3}$ It has been objected to me that this reading begs the metaphysical question (against resultant forces, in particular). But that's wrong: the point is that we can transform any Newtonian models using the principle of composition, regardless of whether or not the real forces are usefully read off of their vectors. As such, the point is also independent of the issues investigated by Lange (2011), which have to do with how the principle is proven and its modal implications.
} 
metaphysically suspect about the fact that different assignments of forces generate the same motions. What worries philosophers is that it seems like we have good, but conflicting, reasons to treat one or another of these models as the "real" one-i.e. the one whose vectors correspond to real forces. In particular, J. Wilson (2009), Moore (2012), and Massin (2017) - borrowing terminology from Cartwright (1980, 1983) - have alleged that we seem to have good reasons to believe in both "component" forces (those that correspond to collisions or attractions) and "resultant" or what I'll call "total" forces. ${ }^{4}$ At least according to the literature, what's really at issue is that the laws of Newtonian physics contain two different types of forces: forces representing particular physical interactions appear in the law of universal gravity and other specific force laws while total forces appear in Newton's second law (see J. Wilson 2009, 526, 532-34; Moore 2012, 361; Massin 2017, 807-11). Since these two different types of forces can be related by the principle of composition, there's an overgeneration problem. As such, the real problem is determining the metaphysical status of the two types of forces; the overgeneration of accelerations provides a motivation for solving this problem.

I have a slightly different diagnosis: behind the two "types" of forces are conflicting metaphysical views about what criteria the real forces of Newtonian physics must meet, or, equivalently, about the conditions under which we can read the real forces off of the force vectors in the mathematical models. Specifically, there's nothing about the second law itself that forces us to read it in terms of total forces; instead, what motivates reading it this way are metaphysical views about the nature of force and acceleration. ${ }^{5}$ The next few paragraphs argue for this conclusion by showing that the features of the physics itself

\footnotetext{
${ }^{4}$ It's worth noting that Cartwright's interests and opinions are different from those tackled in the contemporary discussion. For a detailed discussion of both her complaint and her (changing) views, see Spurret (2001); for an extremely worthwhile response to Cartwright's original worries, see Forster (1988).

${ }^{5}$ Can the same claim be made about the law of universal gravity and physical interactions? I doubt it, but I'll accept that the two "types" of force are analogous in this manner for the sake of argument.
} 
can't be used to motivate the restricted reading.

The first reason that one might think that the second law ought to be read solely in terms of total forces is owed to S. Smith $(2010) .{ }^{6}$ Suppose we have a body $(i)$ gravitationally interacting with two other bodies $(j, k)$. The principle of composition entails that, given initial conditions, we can determine the acceleration of $i$, i.e. $a_{i}$, by determining the distance from $j$ and $k$ to $i\left(r_{i j}\right.$ and $\left.r_{i k}\right)$ and summing the solutions to the following equations:

$$
\begin{aligned}
a_{i j} & =\frac{F\left(r_{i j}\right)}{m_{i}} \\
a_{i k} & =\frac{F\left(r_{i k}\right)}{m_{i}}
\end{aligned}
$$

Since our force function depends on the distance between the bodies, however, the value of $r_{i j}$ and $r_{i k}$ at a time can only be determined by solving the two force functions together. ${ }^{7}$ So, as S. Smith argues, there's a sense in which the effects of the two forces aren't being independently composed: accurately representing the situation requires assuming that both forces are acting on the body at every stage in the process.

But this fact doesn't show that the second law only applies to the total force, i.e. to the vector $F\left(r_{i k}\right)+F\left(r_{i k}\right)$. What it shows is that the evolution of the strength of a force vector over a time depends (in most cases) on what other forces are acting on the body in question, because it depends on things like position in a field. By contrast, the relationship between each individual force vector and the acceleration that it generates remains independent of what other forces are acting on the body, and so the second law can be applied independently to each force vector and then the accelerations summed.

\footnotetext{
${ }^{6}$ The argument from Smith's work is drawn explicitly by Massin (2017); what follows is a reconstruction of what I take this argument to be, not what I take Smith's original conclusion to be. I've transformed S. Smith's harmonic oscillator problem into a gravitational problem. For his discussion, see S. Smith (2010, 361-63); for a discussion of a similar problem, see Moore (2012, 367-68).

${ }^{7}$ I'm simplifying: we'd also need to account for the interaction between $j$ and $k$ and the effect of $i$ on each. The point doesn't depend on these details.
} 
You do have to determine the strength of each force in the conjoined circumstance before applying the second law and the principle of composition-but that doesn't show that the second law must be applied to total forces.

The second argument is that a body cannot have multiple simultaneous accelerations and as such the second law cannot be applied directly to component forces without generating models that are defective because they assign multiple accelerations in this manner (see J. Wilson 2009, 532-34; Massin 2017, 819-20). The problem with this argument is that it is at odds with the face value practice of the science. ${ }^{8}$ Both Newton $(1736 / 1999,426)$ and later Newtonians such as Euler $(1752,175)$ and Lagrange $(1811 / 1997,17,170-71)$ explicitly assign multiple motions (and thus accelerations) to a single body, and Newtonian physics is replete with models that assign multiple acceleration vectors in this manner. We standardly assign both linear and angular acceleration to a single body, for example, even in cases where one and the same force is generating both accelerations. Determining the path of a body affected by such a force is most naturally and commonly achieved by decomposing the single force into the component acting on the body's center of mass and the component that applies only a torque. We then apply (the relevant versions of) Newton's second law to these "component" forces to generate the two different accelerations. Put aside the value of the original intuition momentarily; we can always attempt to deflate the apparent metaphysical implications of the discourse. The point here is just that neither

\footnotetext{
${ }^{8}$ It also doesn't appear to match Newton's historical formulation of the second law in terms of motive forces, though things are much more complicated here because it isn't clear what Newton's version of the second law actually says - it certainly wasn't interpreted by his contemporaries as equivalent to $F=m a$. Euler, whose "Discovery of a New Principle of Mechanics" (1752) popularized $F=m a$, clearly understood this "principle" as distinct from any of Newton's laws; we probably owe the interpretation of the second law in terms of $F=m a$ to Lagrange's Analytical Mechanics, which argues that Euler can be understood as having generalized the second law (Lagrange 1811/1997, 172). There's a substantial historical literature on the subject, which does not, or does not clearly, support the contention that the second law must be understood in terms of total forces. My favored interpretation - offered by Pourciau (2011) - indicates the opposite: Newton's version of the second law ought to be understood in terms of each force acting on a body and only derivatively (in virtue of the principle of composition!) on their total effect.
} 
the technical features of the physics nor the practice thereof give us any reason to adopt the restricted reading of the second law.

The prior point undermines the interpretative arguments of J. Wilson (2009), Moore (2012), and Massin (2017) for reading the second law (only) in terms of resultant forces. One can also find a more deeply metaphysical argument in their work, however (see J. Wilson 2009, 533-34; Massin 2017, 819). An initially plausible version of the argument goes as follows. $F=m a$ can be understood as a bijection between forces and accelerations, and there exists some class of privileged accelerations (the "realized" ones) such that the real forces are the inverse image of this class. Since a body can only have one realized acceleration at a time, the real forces must be the total forces acting on each bodyotherwise the bijection fails. It is legitimate to talk about other forces, but they are merely mathematical fictions.

The argument just given essentially turns on a metaphysical view about how to interpret the science, namely that a force ought to be considered real iff it has a special relationship with a real ("realized") acceleration. A contrasting view is that since Newtonian physics is ultimately about things like collisions, magnetic or gravitational attractions, and other physical interactions in the world, a force ought to be considered real iff it has a special relationship with an interaction. On this view, the real forces ought to be identified with the particularly gravitational (magnetic, etc.) forces found in special force laws, as any other option makes the connection between these interactions and causal efficacy mysterious. It is legitimate to talk about total forces, but these just encode the results of these causally efficacious forces acting jointly.

I think that what really lies behind the alleged overdetermination problem is a conflict between these two different views about the sorts of properties that the real forces of Newtonian mechanics ought to have, not the existence of two different "types" of forces 
in the theory itself. If we accept both views (without the restrictions argued for by Moore 2012 or Massin 2017), then there are too many real forces, and the accelerations of bodies are overgenerated.

\section{Why accelerations aren't a good guide to real forces}

I've claimed that the apparent overdetermination problem that worries philosophers arises not because of the principle of composition but because of conflicting views about how to metaphysically interpret the laws and models of Newtonian physics. In the rest of the paper, I'll consider how these different views align with the technical and historical features of Newtonian science. In this section in particular, I'll examine the view that the real forces can be identified by means of their relation with a class of privileged accelerations, privileged because they are the unique accelerations assigned to each body. My claim is simply that there is no class of accelerations recognized by Newtonian physics that has the properties necessary for the argument presented at the end of the last section to succeed. I begin with those accelerations closest to observation before moving to the most theoretical of these quantities. $^{9}$

The first candidate for privileged accelerations are relative accelerations-i.e., the total acceleration relative to the reference frame of the observer. ${ }^{10}$ If these accelerations are the privileged accelerations, then our criterion demands that the real forces must be equivalent to the observed acceleration of a body multiplied by the mass of that body. This option is unacceptable for the obvious reason that it entails that the real forces vary with the reference frame of the observer. Not only would this imply that causes are never acting

\footnotetext{
${ }^{9} \mathrm{My}$ argument builds on a problem originally raised by A. Wilson (2009), discussed in the next paragraph. The rest of the section demonstrates that the other classes of accelerations that one could privilege fare no better.

${ }^{10}$ Why total? Because any other acceleration will be partial, violating the motivating intuition.
} 
on the observer (since the observer never observes themselves accelerating), it would also imply that what counts as a real cause depends on the perspective of the observer. Neither of these results is acceptable as a characterization of Newtonian physics. ${ }^{11}$

Before we move on, notice that introducing accelerations other than the observed total accelerations - i.e., taking anything other than the observed total accelerations as the privileged class - undermines the intuition that we began with. As noted above, one of the assumptions of the argument is that there can't be multiple accelerations in a body and that the notions of "potential accelerations, non-realized accelerations, or tendencies to accelerate" are somehow nonsensical (Massin 2017, 819; see also J. Wilson 2009, 533-34). As soon we introduce true accelerations, however, we're committed to the two types of accelerations coming apart. As a result, our account will require us to be able to both represent the true accelerations and the relative accelerations simultaneously and explain how the former sum together to generate the latter. Once we take this entirely unproblematic step, however, it's not clear what intuitive or conceptual difficulties are supposed to remain with a view that assigns multiple simultaneous accelerations to a body. Or: given that we need the resources to represent the adding and subtracting of accelerations to account for the relationship between true and relative accelerations, what's preventing us from allowing a body to have multiple true accelerations to begin with? ${ }^{12}$

The second candidate for privileged accelerations are total accelerations relative to a "local" inertial frame, or a reference frame that is (from the point of view of the physics

\footnotetext{
${ }^{11}$ There's nothing wrong with frame-relativity in itself; what's objectionable is the frame-relative interpretation of Newtonian forces. Besides the broad mismatch between this concept and how actual practitioners understood Newtonian physics, understanding forces in a frame-relative manner has the effect of undermining the understanding of inertial frames and true motion in Newtonian physics discussed below: if forces are frame-dependent, they can't be used to define the true motions and thus to distinguish between Tychonic and Keplerian models of the solar system.

${ }^{12}$ It is also dialectically important that Massin $(2017,819)$, at least, takes the fact that "component accelerations" are unobservable theoretical posits as one of the main reasons to doubt their existencebut the same motivation rules out privileging anything beyond relative accelerations as the "actual" or "realized" accelerations.
} 
itself) very nearly an approximation of a true inertial frame (this is the route taken by J. Wilson 2009, 548). The vast majority of calculations within Newtonian physics occur within such frames. There's no need to take into account the influence of the sun's gravity for most terrestrial physics problems, and until very recently there was absolutely no need to take account of the gravitational influence of anything outside of our solar system. Nevertheless, such frames are only approximately inertial, and the total acceleration of a body will change when we shift between them. From the perspective of the surface of Earth, for example, the total acceleration and thus real force acting on a falling body is directed towards the center of the Earth. By contrast, in a frame that takes into account the entire solar system, the total acceleration and real force are directed towards the center of mass of the solar system; the force directed towards the Earth is only a component of this real force. So this option also results in the real causes depending on the choice of frame. ${ }^{13}$ The same problem that we faced earlier has reappeared: the unacceptable frame-relativity of real forces is a consequence of this option as well.

The final option is to wed real forces to the total acceleration in the global inertial frame, i.e., to the total acceleration relative to a body moving with constant velocity relative to the center of mass of the entire universe. Speaking strictly, total accelerations in the global frame never appear in the practice of Newtonian physics; the reference frames employed are always locally inertial at best. On the resulting view, the real causes of the science are items that never appear in any of the equations or models of the science - quite literally everything described in every model is considered to be a fiction. Crucially, the fictional nature of these models doesn't stem from either an incomplete representation of all the causal factors or an idealized presentation of some causes. Instead, it stems from the fact

\footnotetext{
${ }^{13}$ Note that similar considerations undercut J. Wilson (2009)'s experiential argument for total force: the total forces that we experience are total only relative to a particular choice of local inertial frame-we don't experience the total force pulling us towards the sun in the appropriate sense, for example.
} 
that on this view, the local interactions are not actually causal, and thus there's no sense on which giving a local account counts as giving even a partial explanation. From my perspective, in which accuracy to the practice and history of the science is taken as the key desideratum, this is an unappealing result: Newtonians clearly took themselves to giving real explanations with their models, and there doesn't seem to be good reason to deny that they were, at least not on these grounds.

There's also a deeper problem with using the total accelerations in any sort of inertial frame to determine which assignment of forces is real: the relevant notion of acceleration is itself defined in terms of forces. ${ }^{14}$ Newtonian mechanics begins from the assumption that the kinematics of a system-properties like speed, position, and acceleration - vary with the frame of reference. Effectively, this means that there are no intrinsically privileged motions or positions. The particular historical problem raised by this equivalence is that the Copernican and Tychonic models of the solar system cannot be distinguished on the basis of kinematics alone. In order to discriminate between the two models, therefore, there must be an external criterion for "true" motion. Famously, Newton appeals to absolute space as his criterion for true motion, but this gives no grounds for distinguishing between the two models. What does allow him to distinguish between the two models is his operationalization of true motion as that motion that would be observed from the center of force of a system (Newton 1726/1999, 412). ${ }^{15}$ This operationalization of true motion forms the basis of our contemporary understanding of inertial frames, and lies at the heart of Newtonian

\footnotetext{
${ }^{14}$ Notice that if we build gravity into the spacetime structure á la Newton-Cartan theory, we can define a frame-invariant notion of acceleration using the tangent field of the appropriate timelike curve and then define a notion of force on this basis (see Malament 2012, 252-53). This result may appear to belie my claim that the real accelerations are defined in terms of the real forces, but it doesn't: since gravity is encoded in the metric, the metric is playing the role that forces (the forces found in specific force laws!) play in a force-based interpretation, which (to my eyes) just serves to reinforce the point that the relevant notion of acceleration must be defined in terms of whatever is playing that role.

${ }^{15}$ Technically, this requirement is met by any observer moving with a constant velocity relative to the center of force. For our purposes, this caveat can be ignored.
} 
mechanics. What gives us reason to prefer the kinematics of Kepler over those of Tycho is that Newton's dynamics show that the center of force of the solar system is located very near the sun (Newton 1726/1999, 817).

The upshot is the following. If we identify the real forces with those that are equal to the true total acceleration multiplied by the mass, then we're committed to a definitional circularity: true accelerations are defined in terms of their true causes, while the true causes are defined as those that produce the true accelerations. The result is that the very problem that Newtonian mechanics was designed to resolve-discriminating between heliocentric and geocentric models of the solar system - is unsolvable, as there's no way to determine what the true motions are without already knowing the true motions. Notice that this isn't just a practical problem. The outcome here is that one of the most important results of Newtonian science is ruled literally question-begging on the grounds of a particular metaphysical interpretation of what Newton and Newtonians mean by "force." From either a naturalistic viewpoint or just a charitable one, this result is not acceptable.

There's nothing problematic about the idea that $F=m a$ is a bijection between the real forces and some privileged class of accelerations. There's even a good interpretative basis for this idea to be found in the way that Newtonian physics defines true motion. But it can't be used as part of an argument for the claim that total forces are the real ones, as the only class of accelerations that can plausibly play this role are those picked out by applying $F=m a$ to the real forces themselves - and there's no interpretative reason to demand that this class be such that only one such acceleration is assigned to each body. As such, any justification for this restriction must have purely a priori metaphysical grounds. At best, then, views that motivate the reality of total forces by appealing to features of acceleration (as both of J. Wilson 2009, 534 and Massin 2017, 819 explicitly do) are merely compatible with the physics; there aren't good interpretative grounds for taking total forces to be the 
real ones.

I actually think the situation is worse than this, however. Even if we grant that there are a priori metaphysical reasons for thinking that some class of accelerations is so restricted that there can only be one per body, we would need some reason to think that this class of accelerations is the same one picked out by the technical definition of acceleration given by applying $F=m a$ to the real forces. Or: the metaphysical argument for such a restriction cannot turn on pre-theoretical notions of true motions or accelerations, because Newtonian physics offers its own technical understanding of these concepts defined in terms of the true forces. It's hard to see how any metaphysical argument of this sort will get off the ground without reinterpreting these concepts. And if we're committed to reinterpreting these concepts, why not prefer a more robust reinterpretation á la Newton-Cartan?

\section{Why physical interactions are a good guide to real forces}

I've argued that we cannot use the second law and a privileged class of accelerations to motivate a metaphysical account of the real forces. Of course, we can (and must) use observed accelerations in determining which models are empirically adequate, but we can't use accelerations (of any sort) to then determine which force vectors we can read the real forces off of - there's simply no class of accelerations capable of performing this role. In this section, by contrast, I'll argue that a criterion that the real forces should be tied to physical interactions fares much better. Up to this point, our discussion of the physics has focused primarily on issues of predicting accelerations given some model, as this is the primary use of the principle of composition. This isn't the only sort of problem faced in Newtonian physics, however. Newtonian scientists were also concerned with (a) testing and confirming 
Newtonian theory and (b) identifying previously unknown causal influences. ${ }^{16}$ For these purposes, the choice of models is constrained by previously-identified physical interactions such that a force vector can be admitted to the model if and only if it corresponds to one half of the action-reaction pair of a known physical interaction. ${ }^{17}$ The result is that not only is there good reason to think that Newtonians themselves took the forces found in special force laws to be causally efficacious, there's good reasons for us to interpret the science this way as well.

As I remarked above, the principle of composition states that an infinite class of models is empirically equivalent for the purpose of the predicting the motion of a single body. The restriction is crucial - the same set of models will not be equivalent for predicting the motion of other bodies within the system. Newtonian forces are essentially interactive in the sense that for every action on a body there is an equal and opposite reaction by the body on whatever acted on it, and thus any change in the forces acting on a body is acommpanied by a change in the re-actions the body exerts. ${ }^{18}$ When we're modeling a single body, we can separate the action and reaction and focus on one of them, creating an abstraction that we can treat as a vector and thus that we can manipulate according to the principle of composition. But the various models generated in this manner don't (necessarily) indicate the effect of the other part of the interaction on the rest of the system.

An historically important example of exactly this issue can be found in Newton's argument for the inverse-square law, which, strictly speaking, shows that every orbiting body is accelerated towards a body, and whatever forces combine to generate this acceleration are "equivalent to" (in the sense of having the same single-body effect as) a force whose

\footnotetext{
${ }^{16}$ Contemporary scientists who employ Newtonian physics are still concerned with the latter of these projects, of course.

${ }^{17}$ See Forster (1988) for a different way of employing much the same point against Cartwright.

${ }^{18}$ Massin (2017) recognizes this fact and some of the problems that it poses to a view that posits forces where there is nothing for the acted-on body to interact with-i.e., any view that holds that the total forces are causally efficacious.
} 
strength is proportional to the inverse-square of the distance from the focus of the ellipse. In order to move from inverse-square acceleration to universal gravity, it must be the case that the equal and opposite reaction is exercised on the body at this focus - and not on a medium exerting a pressure on the outside of the orbiting bodies (say). Only some of the models capable of generating the effect observed in the motions of the orbiting bodies will have this result. ${ }^{19}$ There are two points here. First, the problem faced by Newton is essentially the same problem raised by contemporary metaphysicians: to determine which of the models, equivalent from the point of predicting the motion of a single body, contains vectors that correspond to the real forces. Second, and in spite of the fact that this is a question contemporary philosophers treat as metaphysical, in Newton's context it has clear empirical implications: different real forces entail different equal and opposite reactions and thus different effects on the rest of the system.

Given the empirical implications, Newton and Newtonian modelers had to find some principled way to discriminate between these models, to determine which ones we can reliably read the real causes off of. They identified such models by comparing them to (and later, constructing them on the basis of) identifiable physical interactions, such as collisions or gravitational attractions between massive bodies. In practice, this method means reading the real forces off of a model if and only if every force vector corresponds to one half of the action-reaction pair of a known physical interaction. Objects don’t move without being acted on, and so there aren't force vectors without corresponding interactions. And this, in turn, is essentially the same intuition that we identified as motivating the view that the

\footnotetext{
${ }^{19}$ Since I'm primarily interested in the implications of the third law, I'm passing over the question of whether Newton actually provided a sufficient argument for his solution. But note: while this is a particularly thorny question for Newton scholarship, precisely the issue for Newton's contemporaries - such as Cotes and Huygens - is that the mathematical models employed to demonstrate the inverse-square rule don't discriminate between attractions and impulses, and this difference has physical consequences. For detailed discussion of both this issue and the derivation more broadly, see Stein (1990), G. E. Smith (2002), and Harper (2012, 346-55).
} 
real forces are those found in the special force laws.

This use of physical interactions is especially apparent in post-Newton modeling of the solar system, as testified to by both historical scientists such as Somerville (1831, xi) and Herschel $(1830 / 1966,201)$ and contemporary work by G. E. Smith $(2014) .{ }^{20}$ Consider the work of Urbain le Verrier, who famously postulated the existence of Neptune on the basis of perturbations in the orbit of Uranus and, more infamously and less successfully, postulated the existence of Vulcan on the basis of perturbations in the orbit of Mercury. In both cases, le Verrier's argument was essentially as follows. Using known physical interactions -i.e. gravitational relationships between massive bodies - and the laws of Newtonian mechanics, we can construct a model of the solar system. This model predicts motions that deviate from observation in a systematic manner-there's a leftover pattern in the phenomena not attributable to any interaction between such bodies. Because forces exist only where physical interactions do, we can infer that either the prior measurement of the strength of an interaction is wrong (and in a systematic manner) or that there is an additional interaction not accounted for by the model.

In the case of Uranus, the additional interaction was found quite quickly. In the case of Mercury, the additional "interaction" cannot be modeled within Newtonian mechanics, but the physical interaction constraint was essential even in this case. As Simon Newcomb pointed out in 1882, le Verrier's Vulcan hypothesis has two defects. First, no such planet has ever been observed. Second, the forces that the Vulcan hypothesis requires are impossible as no planet or group of planets "could exist and produce the observed effect without disturbing the secular motions of the node of Mercury and Venus" (Newcomb 1882, 475). In other words, no planet could possibly be exerting the required force on Mercury without also interacting with other bodies in ways that would disrupt the entire model.

\footnotetext{
${ }^{20}$ The next two paragraphs follow G. E. Smith (2014).
} 
The type of reasoning here exhibited by le Verrier and Newcomb is essential to the practice of Newtonian astronomy in two ways that are important for our discussion. First, insofar as the models of le Verrier, Newcomb, and other astronomers served to test Newton's theory of gravity - and since they revealed the infamous discrepancy in Mercury's precession, they deserve that characterization - such models must admit forces if and only if there is an identifiable physical interaction to tie them to. This constraint came to the fore most notably in the precession of Mercury's perihelion, where the observed accelerations are ultimately only predicted by models that include force vectors for which there are no identifiable interactions, and this provides crucial evidence that Newtonian physics is incorrect. Without the metaphysical connection between physical interactions and real forces, the models wouldn't be constrained in the same way, and so Newtonian theory would have no in-principle problem with accounting for the observed accelerations of Mercury.

Second, as G. E. Smith (2014) emphasizes, in order for the deviations from a model to be successfully used as evidence for some not-yet identified or measured interaction à la Neptune or Vulcan, the model has to screen out the influences that it claims to represent. We infer the existence of unseen planets - and now, objects like black holes - on the basis of deviations between the model built on what we already know and observed accelerations. Such deviations are only informative if all the force vectors in the model correspond to real causes; otherwise, such deviations would be entirely meaningless, the result of a bad curvefit. Once again, the connection between forces and physical interactions plays a crucial role here: we're inferring the existence of an additional physical interaction, and so the previously identified forces must be identified with interactions as well.

Allow me to return now to the dialectic where we left it at the end of $\S 1$. There's a conflict between different views about how to interpret the physics: one that holds that our account of the real forces must be constrained by their relationship to a privileged class 
of accelerations and another that instead ties the real forces to physical interactions. In $\S 2$, I argued that the first of these views breaks down on inspection. Newtonian physics simply doesn't provide any privileged class of accelerations capable of playing this role. In this section, I've argued that the other view - that the real forces are to be tied to physical interactions like collisions and gravitational attractions - has a much better fit: not only does it play a role in Newtonian practice, this interpretation of real forces does significant heavy lifting in the confirmation of Newtonian theory and the discovery of new causal influences. There's good reason, therefore, to see the forces most directly tied to physical interactions between bodies - namely, those that figure in the special force laws - as the real forces of Newtonian physics. It's these - and not the total force acting on a body - that have causal implications of their own.

\section{Conclusion}

I've argued for three main theses in this paper. First, the apparent overdetermination problem results from conflicting intuitions about how to interpret Newtonian mechanics rather than a conflict within the physics itself. Second, there is no privileged class of accelerations that can be used to determine which forces are real, and thus there's no interpretative reason to prefer a metaphysical picture in which total forces are real. Third, not only can identifiable physical interactions such as collisions be used to determine which forces are real, they in fact are used this way, and in an apparently ineliminable way. Given the assumption that a force-based interpretation of Newtonian mechanics is the right one, the consequence is that forces that directly represent physical interactions are the real causes of Newtonian physics. Once we recognize that only one of the two metaphysical interpretations is motivated by the formulation and practices of Newtonian mechanics, any interpretative grounds for the overdetermination problem also disappear. 
That's not to say that all of the issues in this area have been resolved. While I'm skeptical of the soundness of the a priori metaphysical and experiential arguments employed by J. Wilson (2009), Moore (2012), and Massin (2017), I haven’t shown that these arguments are unsuccessful. To my mind, the more interesting issues concern whether the arguments that I've presented generalize. In this paper, I've primarily considered $18^{\text {th }}$ century Newtonian foundations and the role of physical interactions when the forces at issue are gravitational. Switching our attention from Newton's force laws to the $19^{\text {th }}$ century mechanics of conservation and least-action principles, or to different kinds of forces such as shear stresses, might also require us to switch our metaphysical understanding of forces. As M. Wilson (2006) has stressed, classical physics is extraordinarily multi-faceted - these other facets might allow for, or even demand, different conceptions of force.

That said, the aspects of Newtonian physics that I've surveyed are unequivocal in the following respect. Insofar as Newtonian mechanics refers to an historical discipline with a force-based metaphysics that still has some explanatory and pedagogical usefulness (J. Wilson 2007, 176), there are causally efficacious elements in the metaphysics of this science - namely, the real forces. At least in the case of gravity, these are tied directly to physical interactions; they aren't abstractions like the "total force" acting on a body. Not only does the science provide no reason for believing that the latter are causally efficacious, they clearly tell against it: if the total forces are frame-relative quantities, they cannot play the roles played by causally efficacious forces in various applications of Newtonian science; if they they aren't, the science seems to give them no causal role. There are, therefore, no good interpretative reasons for thinking that the composition of forces presents us with an overdetermination problem. There are, moreover, good reasons to think that the forces found in the special force laws are not just what Newton meant by "forces in a true and physical sense" (Newton 1726/1999, 408), they're the best candidates for the causally 
efficacious forces of the science itself.

\section{Acknowledgments}

I would like to thank Nic Teh; Katherine Brading; James Nguyen; Evan Arnet; audiences at Bloomington, Leeds, and Notre Dame; and a number of anonymous reviewers for helpful comments on earlier drafts of this paper. I am additionally indebted to George E. Smith for his invaluable instruction on Newton and Newtonian mechanics, without which I would not have been able to write this essay.

\section{References}

Cartwright, N. (1980). Do the laws of physics state the facts? Pacific Philosophical Quarterly, 61(1/2), 75-84.

Cartwright, N. (1983). How the laws of physics lie. Oxford: Oxford University Press.

Creary, L. (1981.) Causal explanation and the reality of natural component forces. Pacific Philosophical Quarterly, 62(2), 148-57.

Euler, L. (1752). Decouverte d'un Nouveau Principe de Mechanique [Discovery of a New Principle of Mechanics]. Mémoires de l'académie des sciences de Berlin, 6, 185-217.

Forster, M. (1988). Unification, explanation, and the composition of causes in Newtonian mechanics. Studies in the History and Philosophy of Science, 19(1), 55-101.

Harper, W. (2012). Isaac Newton's scientific method: Turning data into evidence about gravity and cosmology. Oxford: Oxford University Press. 
Herschel, J. (1830/1966). Preliminary discourse on the study of natural philosophy. New York: Johnson Reprint Corporation.

Lagrange, J-L. (1811/1997). Analytical mechanics, ed. and trans. Boissonnade, A and Vagliente, V. N. Dordrecht: Kluwer.

Lange, M. (2011). Why do forces add vectorially? A forgotten controversy in the foundations of classical mechanics. American Journal of Physics, 79(4), 380-88.

Malament, D. (2012). Topics in the foundations of general relativity and Newtonian gravitation theory. Chicago: University of Chicago Press.

Massin, O. (2017). The composition of forces. The British Journal for Philosophy of Science, $68(3), 805-46$.

Moore, D. (2012). A nonreductive model of component forces and resultant force. International Studies in the Philosophy of Science, 26(4), 359-380.

Newcomb, S. (1882). Discussion of observed transits of Mercury, 1677-1881. In his Astronomical papers, prepared for the use of the American Ephemeris and Nautical Almanac, Vol. 1. (pp. 363-487). Washington: Bureau of Navigation.

Newton, I. (1726/1999). Mathematical principles of natural philosophy, trans. Cohen, I. B. and Whitman, A. Berkeley, CA: University of California Press.

Pourciau, B. (2011). Is Newton's second law really Newton's? American Journal of Physics, $79(10), 1015-22$.

Smith, G. E. (2002). From the phenomenon of the ellipse to an inverse-square force: Why not? In Malament, D. (ed.) Reading natural philosophy: Essays in the history and philosophy of science and mathematics. (pp 31-70). La Salle, IL: Open Court. 
Smith, G. E. (2014). Closing the loop: Testing Newtonian gravity, then and now. In Beiner, Z. and Schliesser, E. (eds.) Newton and empiricism. (pp. 262-351). Oxford: Oxford University Press.

Smith, S. (2010). Elementary classical mechanics and the principle of the composition of causes. Synthese, 173(3), 353-73.

Somerville, M. (1831). Mechanism of the heavens. London: John Murray.

Spurrett, D. (2001). Cartwright on laws and composition. International Studies in the Philosophy of Science, 15(3), 253-68.

Stein, H. (1990). "From the phenomena of motions to the forces of nature": Hypothesis or deduction? PSA: Proceedings of the Biennial Meeting of the Philosophy of Science Association, 1990(2), 209-22.

Wilson, A. (2009). Disposition-manifestations and reference-frames. Dialectica, 63(4), 591601.

Wilson, J. (2007). Newtonian forces. British Journal for the Philosophy of Science, 58(2), 173-205.

Wilson, J. (2009). The causal argument against component forces. Dialectica, 63(4), 525-54.

Wilson, M. (2006). Wandering significance. Oxford: Oxford University Press. 